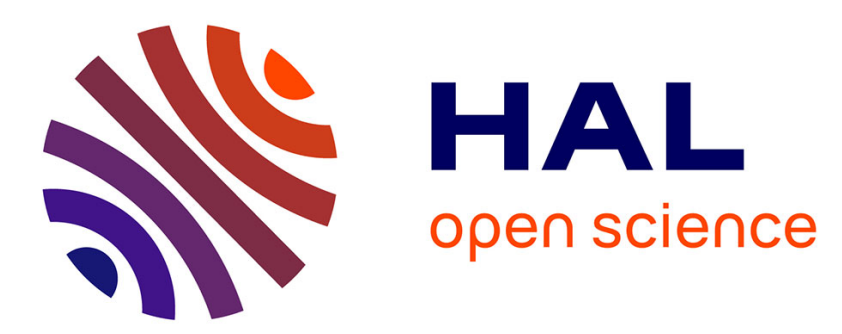

\title{
Air traffic control radar interference event in the Galileo E6 band: Detection and localization
}

\author{
Javier Arribas, Jordi Vilà-Valls, Antonio Ramos, Carles Fernández-prades,
} Pau Closas

\section{- To cite this version:}

Javier Arribas, Jordi Vilà-Valls, Antonio Ramos, Carles Fernández-prades, Pau Closas. Air traffic control radar interference event in the Galileo E6 band: Detection and localization. Navigation, 2019, 66 (3), pp.505-522. 10.1002/navi.310 . hal-03203904

\section{HAL Id: hal-03203904 \\ https://hal.science/hal-03203904}

Submitted on 21 Apr 2021

HAL is a multi-disciplinary open access archive for the deposit and dissemination of scientific research documents, whether they are published or not. The documents may come from teaching and research institutions in France or abroad, or from public or private research centers.
L'archive ouverte pluridisciplinaire HAL, est destinée au dépôt et à la diffusion de documents scientifiques de niveau recherche, publiés ou non, émanant des établissements d'enseignement et de recherche français ou étrangers, des laboratoires publics ou privés. 


\section{OATAO \\ Open Archive Toulouse Archive Ouverte}

\section{Open Archive Toulouse Archive Ouverte (OATAO)}

OATAO is an open access repository that collects the work of some Toulouse researchers and makes it freely available over the web where possible.

This is an author's version published in: https://oatao.univ-toulouse.fr/27101

Official URL:https://doi.org/10.1002/navi.310

\section{To cite this version :}

Arribas, Javier and Vilà-Valls, Jordi and Ramos, Antonio and Fernández-Prades, Carles and Closas, Pau Air traffic control radar interference event in the Galileo E6 band: Detection and localization. (2019) Navigation, 66 (3). 505-522. ISSN 0028-1522

Any correspondence concerning this service should be sent to the repository administrator: 


\title{
Air traffic control radar interference event in the Galileo E6 band: Detection and localization
}

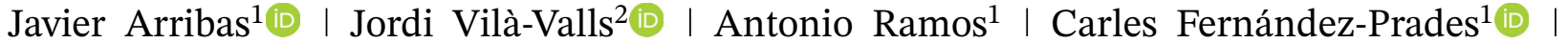 \\ Pau Closas ${ }^{3}$
}

${ }^{1}$ Statistical Inference for Communications and Positioning Department, Centre Tecnològic de Telecomunicacions de Catalunya (CTTC/CERCA), Barcelona, Spain

${ }^{2}$ ISAE-SUPAERO, University of Toulouse, Toulouse, France

${ }^{3}$ Electrical and Computer Engineering Department, Northeastern University, Boston, Massachusetts

\section{Correspondence}

Javier Arribas, Statistical Inference for

Communications and Positioning

Department, Centre Tecnològic de

Telecomunicacions de Catalunya

(CTTC/CERCA), 08860, Barcelona, Spain.

Email: jarribas@cttc.es

\section{Funding information}

Generalitat de Catalunya, Grant/Award Number: 2017-SGR-1479; Ministerio de Economía y Competitividad, Grant/Award Number: TEC2015-69868-C2-2-R (ADVENTURE); National Science Foundation (NSF) under Grant/Award Numbers: CNS-1815349 and ECCS-1845833

\begin{abstract}
The Galileo E6 band operates at a nonprimary frequency band within the L-band. This presents challenging situations in the vicinity of other, legitimate, radiolocation services. This is the case for Air Traffic Control (ATC) radar, which is seen as an in-band-pulsed interference by a GNSS receiver. This paper provides a detailed study of the impact of such interference, as well as localization approaches. Particularly, the paper describes the ATC jamming event captured on a GNSS permanent station, its effects on a real receiver, and how it was tracked to localize the source of interference. An ultra low-cost array-based solution is prototyped, based on commercial off-the-shelf devices, that implements a two-element array. Experimental results are shown and discussed using real data, validating the localization performance of the prototype.
\end{abstract}

\section{1 | INTRODUCTION}

The vulnerability of Global Navigation Satellite Systems (GNSS) to Radio Frequency Interferences (RFI), either intentional or unintentional, is a fact widely studied in recent times. ${ }^{1}$ The performance degradation and even the denial-of-service situations are especially important for safety-critical operations, such as aeronautical high-accuracy positioning and navigation or for distributed timing services, where availability, integrity, and accuracy are mandatory. One of the most powerful unintentional interference sources comes from radionavigation aids. In particular, the civil air traffic control (ATC) primary and secondary surveillance radars operated in the L-Band (1250 to $1350 \mathrm{MHz}$ ) are very close to the Galileo E5 (1189 MHz) and GPS L5 (1207.140 MHz) bands. Because of their high power-pulsed transmissions, which can reach values of tens of kilowatts, they are disrupting the GNSS service, as reported in previous studies. ${ }^{2-5}$ The ATC interference in the Galileo E5 and GPS L5 bands represents an out-band interference that can be mitigated, for instance, using sharper bandpass filters in the receiver front-end. ${ }^{4}$

In February 2017, the European Commission and the European GNSS Agency (GSA) confirmed that the first generation of Galileo satellites would already provide users with high accuracy and authentication services on the 
Galileo E6 band (1260 to $1300 \mathrm{MHz}$ ), referred to as Galileo commercial service (CS). ${ }^{6}$ The ATC radar interference is particularly due to the following facts:

- The Galileo E6 band is completely overlapped with the ATC L-Band, which produces a strong-pulsed in-band interference that cannot be mitigated using an antenna filter. This is especially problematic if the receiver uses a multi-band antenna (such as E1/L1 + E5/L5 + E6).

- The current International Telecommunication Union (ITU) regulations ${ }^{7}$ and the European radiofrequency spectrum regulation consider radionavigation aids and the GNSS service as co-primary users. However, in the European Conference of Postal and Telecommunications Admistrations (CEPT), ${ }^{8}$ it is recommended that GNSS shall not interfere with radionavigation aids (see note 5.329), which provides the latter with higher priority.

- The existing legacy ATC L-Band radars, which should be progressively migrated to the S-Band, are still in their medium lifespan.

The confluence of these factors guarantees the persistence of the interference for several years in the future. The authors detected such a situation in Spain, in the Barcelona metropolitan area during their daily research activity. The same problem is likely to occur in other locations all over Europe, representing a real threat that needs to be seriously taken into account. This contribution reports a real ATC radar interference and its impact on GNSS receivers. Together with the detection and analysis of the unintentional ATC radar jamming, an ultra low-cost array-based solution for interference localization is proposed, which is composed of two elements that estimate azimuth of the interference signal. Notice that in addition to the results provided in this article, the mitiga- tion of such interference can be easily implemented via a real-time pulse blanking algorithm, ${ }^{9}$ as was done in a previous contribution by the same authors, ${ }^{10}$ where a single antenna setup and an open source software-defined GNSS receiver ${ }^{11}$ were considered.

The paper is organized as follows. Section 2 describes an ATC radar interference event detected in the Barcelona metropolitan area that was reported by the authors to the national radio frequency management authorities due to its critical impact in GNSS. Section 3 adresses the impact and mitigation of the ATC radar interference. Section 4 briefly discusses the antenna array signal model for an arbitrary interference waveform, describing an interference detection algorithm and a signal direction of arrival (DOA) estimation method. Section 5 proposes a maximum likelihood (ML) estimation-based triangulation algorithm for interference location, using a set of DOA estimations. The implementation of a prototype based on software-defined radio (SDR) technology is described in Section 6, including synchronization challenges in low-cost devices. Section 7 shows the algorithm implementation validation by simulations, and Section 8 shows the results obtained in a real-life measurement campaign to detect and localize the ATC radar-pulsed interference. Finally, Section 9 concludes the paper.

\section{2 | ATC RADAR INTERFERENCE EVENT}

In February 2016, the GESTALT ${ }^{\circledR}$ (GNSS SignAL Testbed) lab facility (see Arribas et $\mathrm{al}^{12}$ for a detailed description of the GNSS hardware and software set) located at the Centre Tecnològic de Telecomunicacions de Catalunya (CTTC) headquarters in Castelldefels (Barcelona, Spain) was used to capture and analyze the new Galileo E6 signals

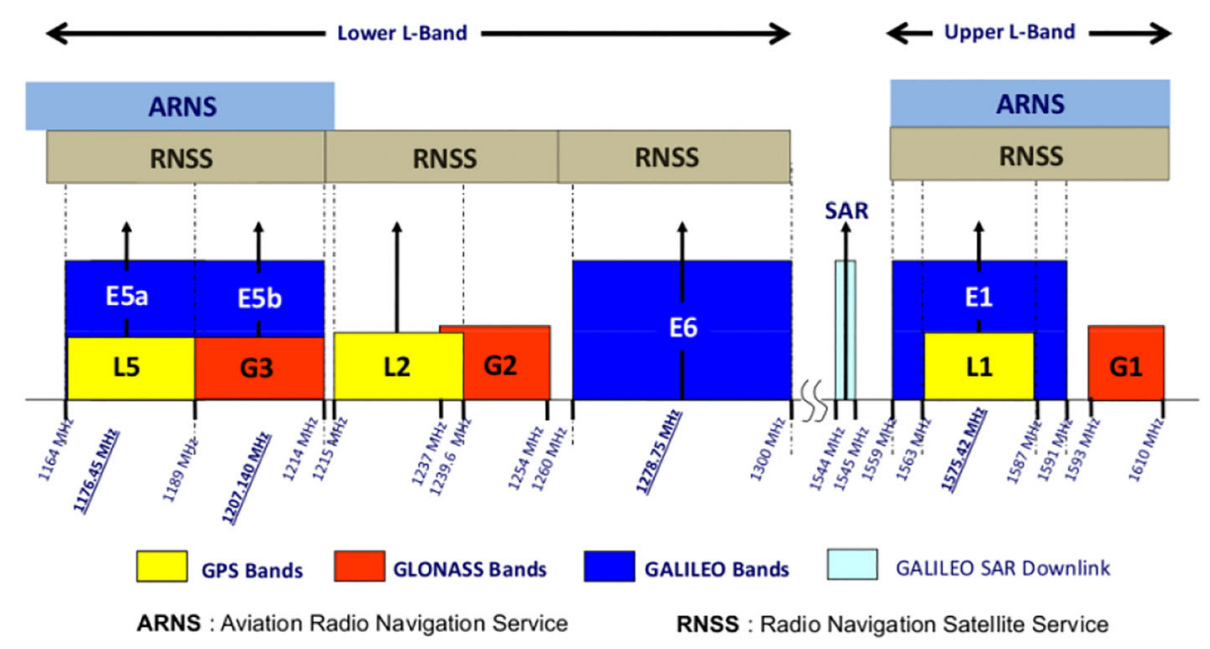

FIGURE 1 GNSS band frequency allocation (source: http://www.navipedia.net/) [Color figure can be viewed at wileyonlinelibrary.com and www.ion.org] 


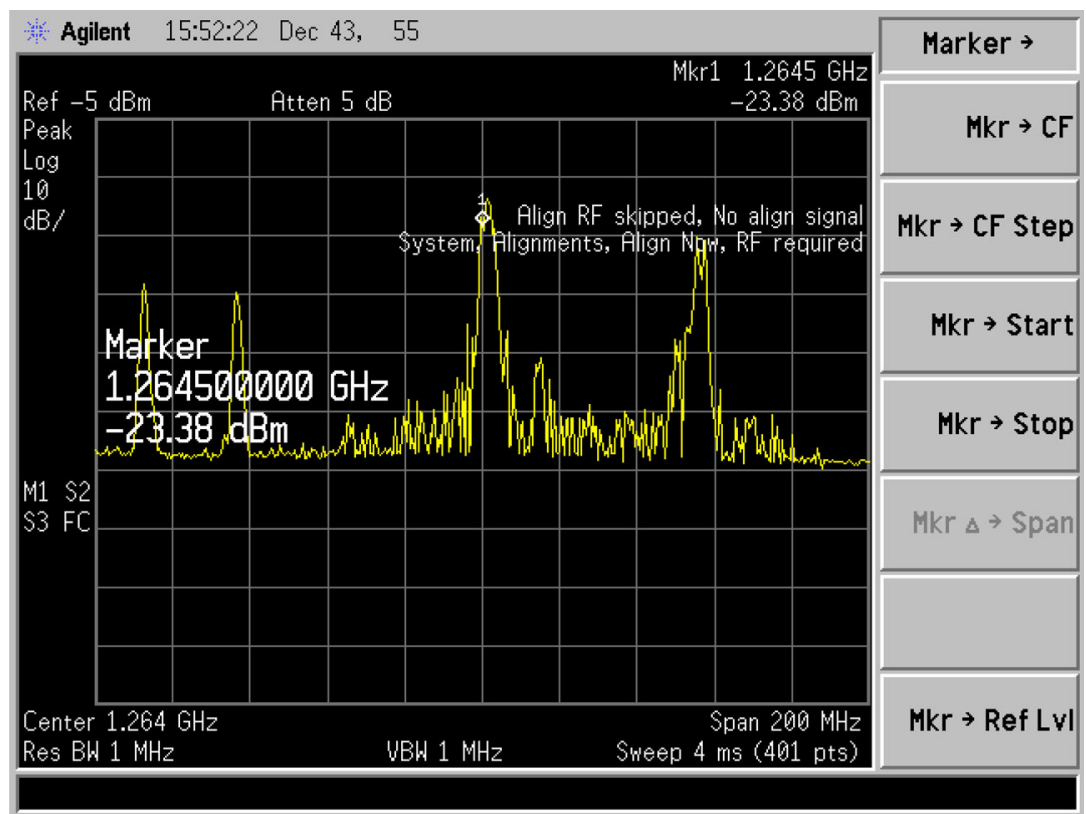

FIGURE 7 Spectrum detail of the $1.2655 \mathrm{GHz}$ interference [Color figure can be viewed at wileyonlinelibrary.com and www.ion.org]

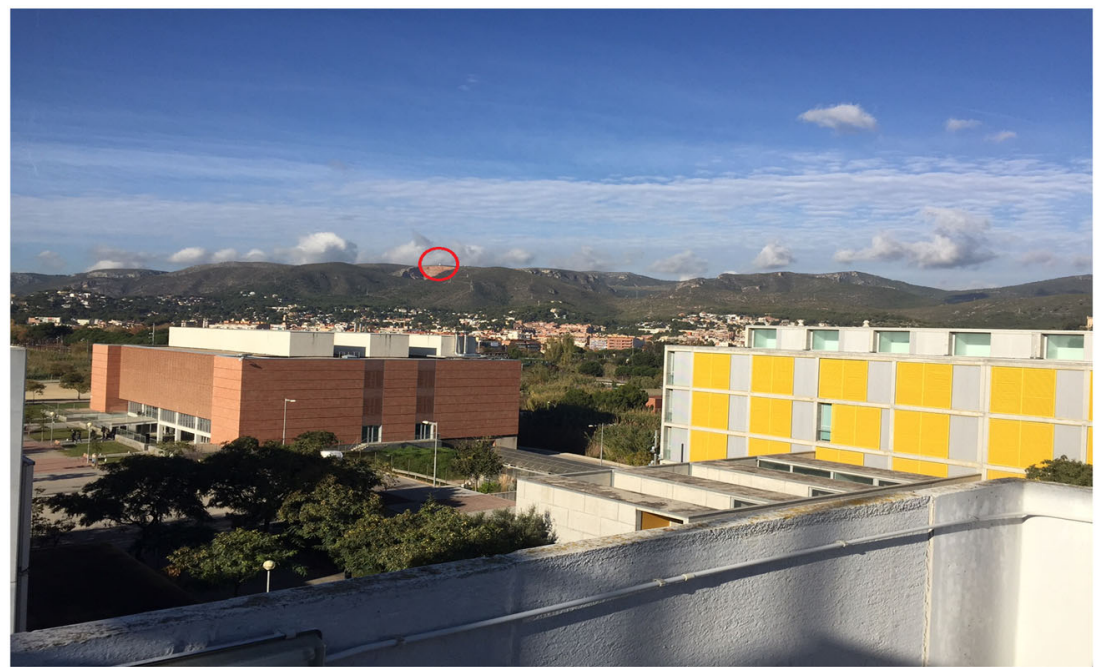

FIGURE 8 Barcelona L-band air traffic control (ATC) primary radar in the Global Navigation Satellite Systems (GNSS) receiver line of sight [Color figure can be viewed at wileyonlinelibrary.com and www.ion.org]

filters. Even by tuning the front-end to the L1/E1 band, it does not protect the input amplifiers and mixers from saturation due to the interference power. The effects are new intermodulation products, which affect all GNSS bands, thus, the out-of-band interference becomes an in-band interference. SDR receivers are commonly connected to such front-ends, and thus it is specially important to assess the interference impact to SDR GNSS receivers and implement countermeasures to minimize such interference effects, as reported in Arribas et al. $^{10}$ For completeness, we provide such impact and mitigation analysis in the following section.

\section{I IMPACT AND MITIGATION OF THE ATC RADAR INTERFERENCE IN SDR GNSS RECEIVERS}

As already stated, when using SDR GNSS receivers in the L1/E1 band with general purpose front-ends, the ATC 

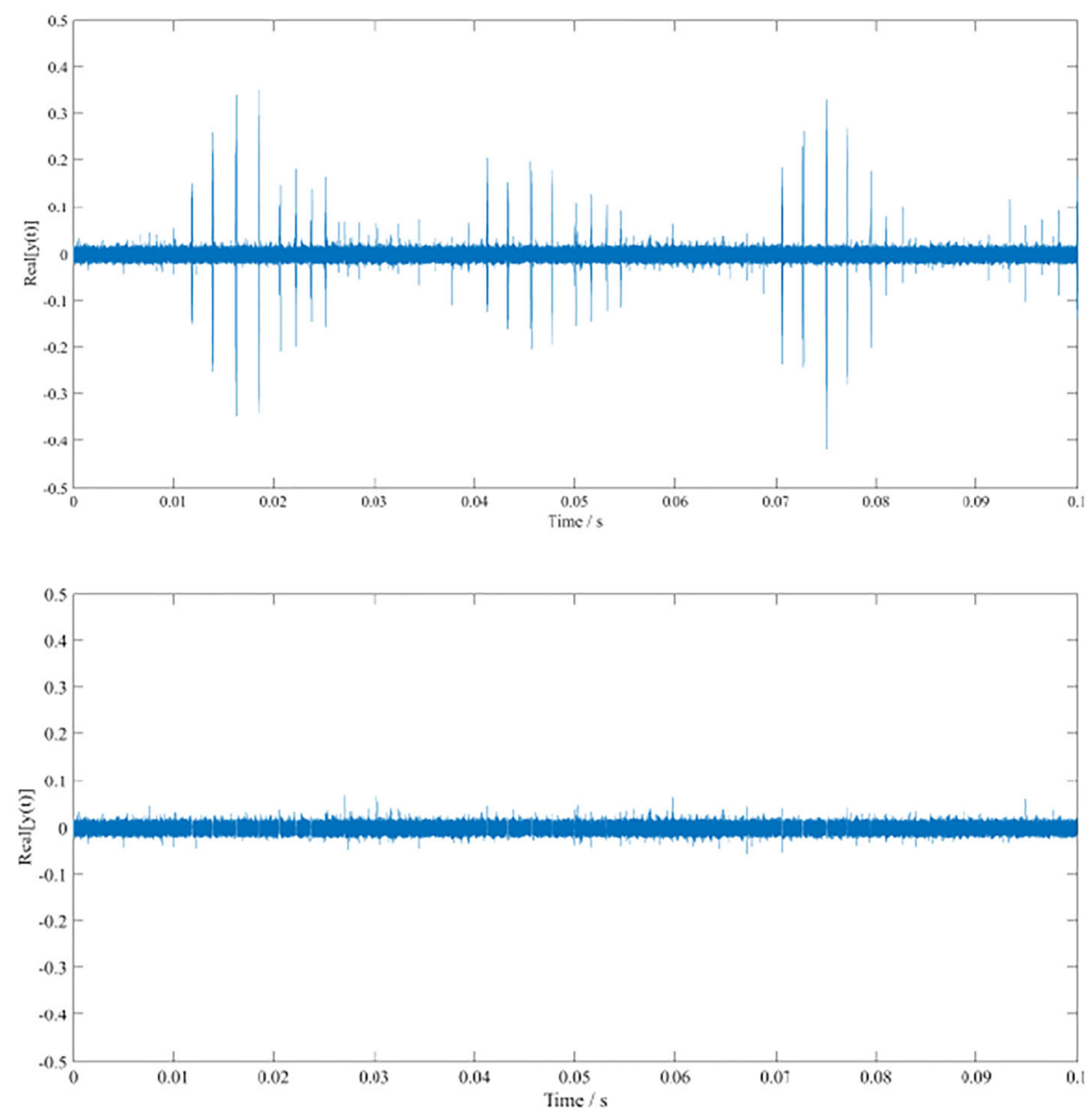

FIGURE 9 Galileo E1 signal interfered by ATC radar pulses (top), and the same input signal after pulse blanking (bottom) [Color figure can be viewed at wileyonlinelibrary.com and www.ion.org]
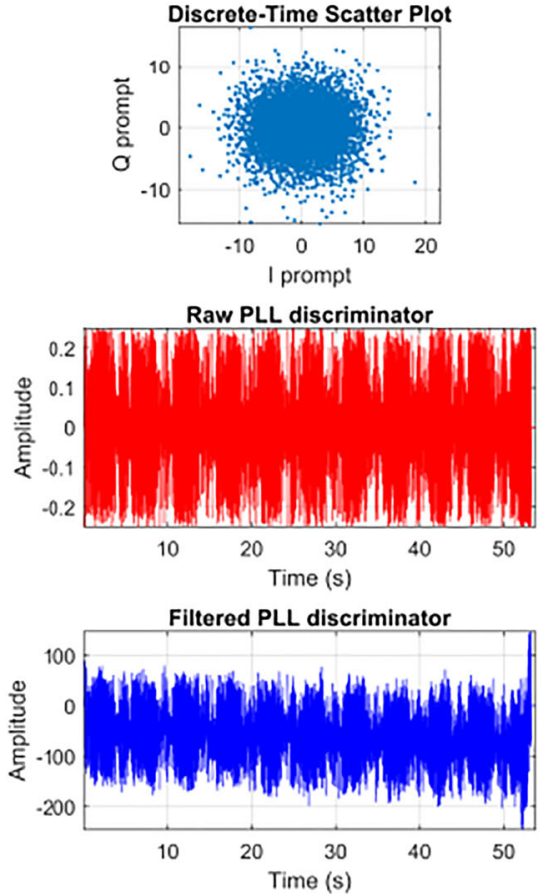
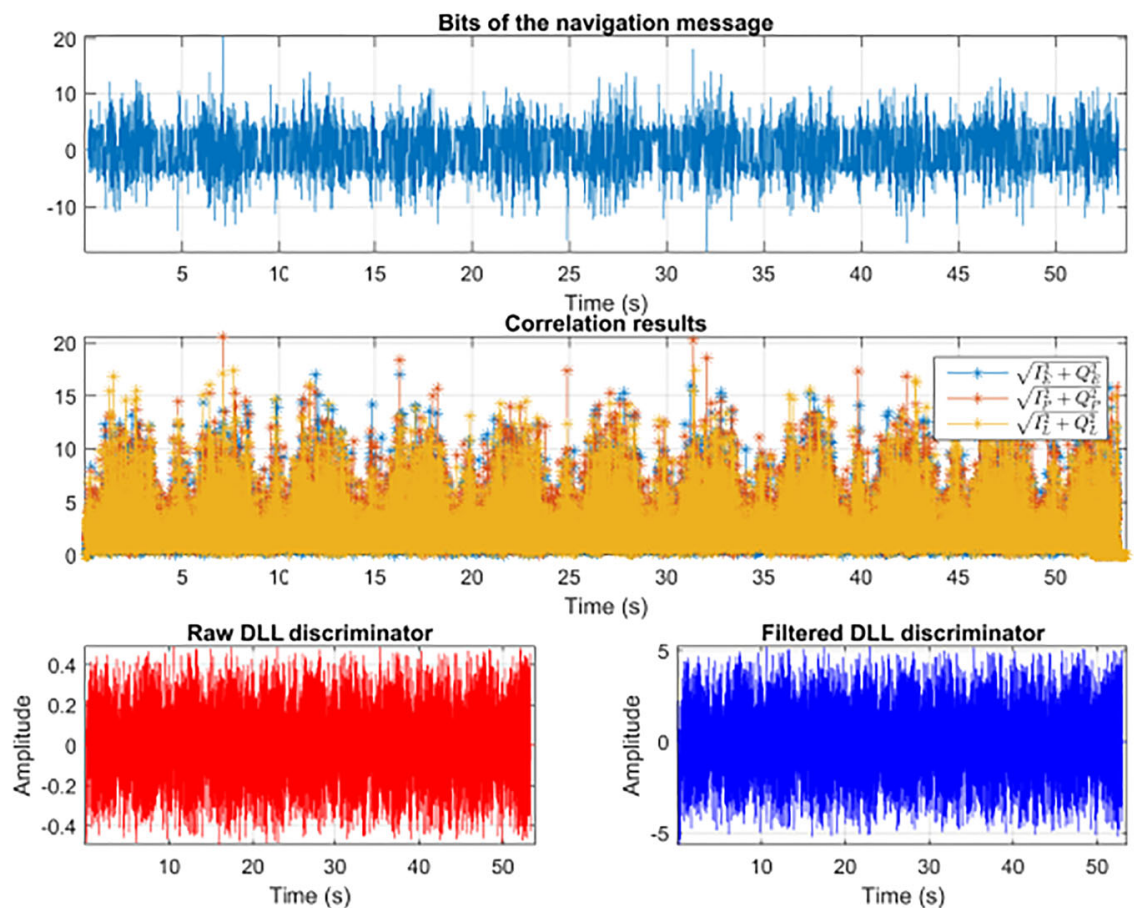

FIGURE 10 Tracking results with the open source GNSS-SDR receiver for Galileo E1 under radar interference [Color figure can be viewed at wileyonlinelibrary.com and www.ion.org] 

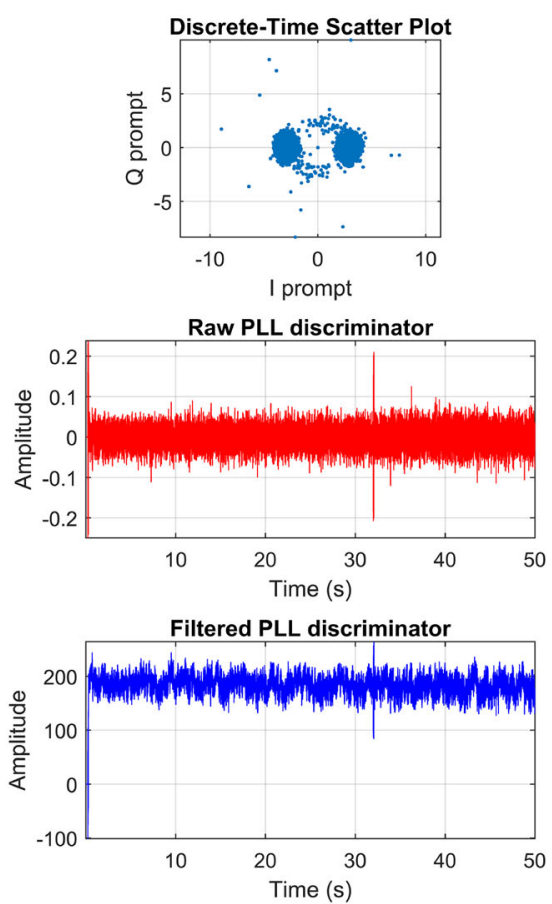

Bits of the navigation message

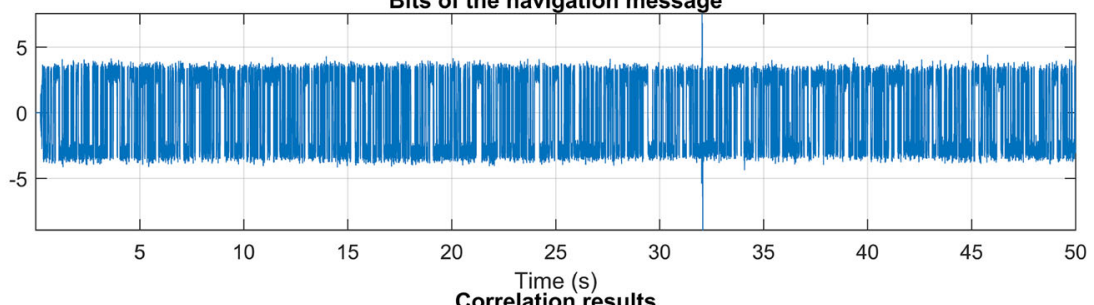

Correlation results
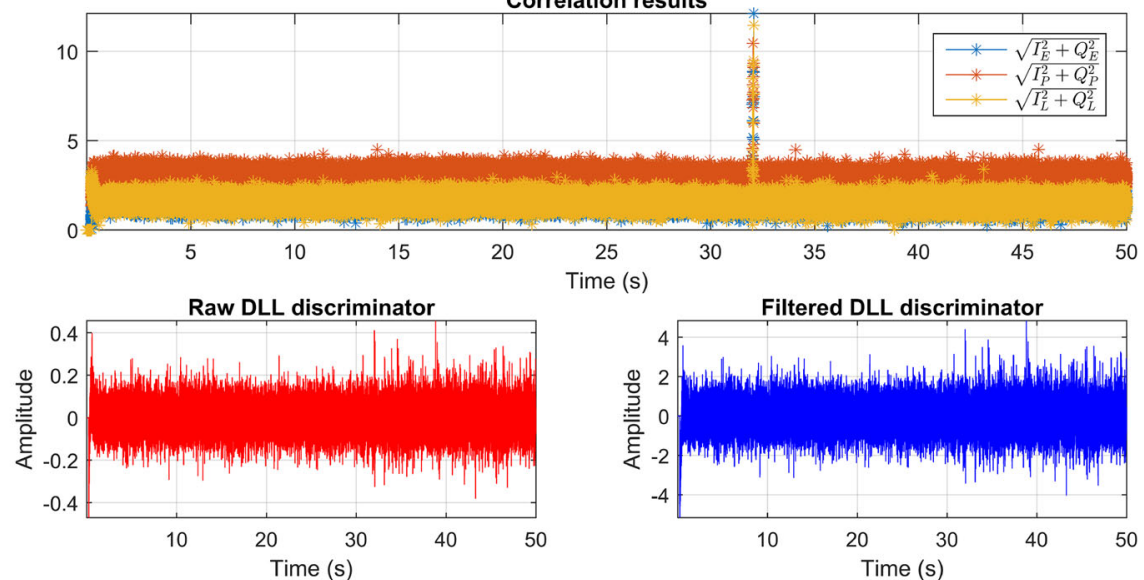

FIGURE 11 Tracking results with the open source Global Navigation Satellite System software-defined radio (GNSS-SDR) receiver for Galileo E1 under radar interference, considering a pulse blanking filter with probability of false alarm $=0.04$ [Color figure can be viewed at wileyonlinelibrary.com and www.ion.org]

radar interference in the E6 band may appear as an out-of-band interference. This is clear from the top plot in Figure 9 for a Galileo example, where the out-of-band pulses are leaking from E6 to E1. These radar pulses cause spurious correlation spikes at the acquisition and tracking stages of the receiver.

To assess the impact of the real ATC radar interference into an SDR GNSS receiver, we consider the use of an open source software defined GNSS receiver. ${ }^{11}$ We show the tracking results for 53 seconds of signal in Figure 10. In this case, the receiver is not able to correctly track the signal (continuous loss-of-lock) because the pulses cause spikes in the early-prompt-late correlators. The top plots in Figure 10 show that the navigation data bits (BPSK) are not decoded, thus the receiver is not able to provide a PVT solution due to the radar interference, thus a complete GNSS service denial results.

An easy countermeasure for such interference is to use a pulse blanking algorithm. ${ }^{9}$ An example of the blanked signal is shown in the bottom plot in Figure 9, where the pulses have been effectively filtered. Using the blanking filter considerably improves the signal quality.

The tracking results considering a real-time pulse blanking filter with a false alarm of 0.04 are shown in Figure 11. In this case, the navigation message is correctly decoded (see the BPSK data bits in the top plots) and the receiver provides a PVT solution, with a time to first fix of 42 seconds. Only one pulse at second 32 is not blanked, but this does not break the correct receiver operation. The present contribution extends the preliminary analysis in Arribas et $\mathrm{a}^{10}$ and proposes a low-cost antenna array prototype made with off-the-shelf components and SDR tools, with the main goal being to gather DOA measurements of the interference source, which will be used in the DOA-based localization algorithm.

\section{4 | INTERFERENCE DETECTION AND DOA ESTIMATION}

Considering that a GNSS interference signal is received with an $N$-element antenna array, the discrete baseband signal model can be defined as

where:

$$
\mathbf{X}(t)=\mathbf{h} \mathbf{d}_{\text {int }}(t)+\mathbf{N}(t),
$$

- $\mathbf{X}(t)=\left[\mathbf{x}\left(t-(K-1) T_{s}\right), \ldots, \mathbf{x}(t)\right] \in \mathbb{C}^{N \times K}$ is referred to as the space-time data matrix, where $\mathbf{x}(t)=\left[x_{1}(t) \ldots x_{N}(t)\right]^{T}$ is defined as the antenna array baseband snapshot; each row corresponds to one antenna and $K$ is the number of captured snapshots. The snapshot time interval can be defined as $T_{\text {snap }}=K T_{S}$ where $F_{S}=1 / T_{S}$ is the sampling frequency.

- $\mathbf{h}=\left[h_{1}, \ldots, h_{N}\right]^{\top} \in \mathbb{C}^{N \times 1}$ is the non-structured channel model, which includes both the channel and the array response. The channel vector assumes the role of the spatial signature but does not impose any structure. The 
arbitrary structure of $\mathbf{h}$, which is considered constant during $T_{\text {snap }}$, is not only parameterized by the interference signal DOA and the location of antennas, but also may include other unmodeled phenomena.

- $\mathbf{d}_{\text {int }}(t)=\left[s\left(t-(K-1) T_{s}-\tau\right), \ldots, s(t-\tau)\right] \in \mathbb{C}^{1 \times K}$ is the discrete version of the interference signal with an arbitrary waveform, such as the pulsed interference shown in Figure 5.

- $\mathbf{N}(t)=\left[\mathbf{n}\left(t-(K-1) T_{s}\right) \ldots \mathbf{n}(t)\right] \in \mathbb{C}^{N \times K}$ is a complex, circularly symmetric Gaussian vector process with zero-mean, temporally white, and spatially white assuming:

$$
\begin{gathered}
\mathbb{E}\left\{\mathbf{n}\left(t_{n}\right)\right\}=\mathbf{0}, \\
\mathbb{E}\left\{\mathbf{n}\left(t_{n}\right) \mathbf{n}^{\top}\left(t_{m}\right)\right\}=\mathbf{0}, \\
\mathbb{E}\left\{\mathbf{n}\left(t_{n}\right) \mathbf{n}^{H}\left(t_{m}\right)\right\}=\sigma^{2} \mathbf{I},
\end{gathered}
$$

where $\mathbb{E}\{\cdot\}$ is the expectation operator, it is assumed that the noise has double-sided spectral density $\sigma^{2}=\frac{N_{0}}{2}$ $\mathrm{W} / \mathrm{Hz}$, and I stands for the identity matrix.

For the purpose of this study, and due to the low GNSS signal power available at Earth's surface, GNSS signals can be assumed to be well below the noise floor and, therefore, their contribution can be considered as Gaussian noise and included in the thermal noise term. ${ }^{14}$

The first step of an interference localization system consists of determining if the interference is present inside the receiver's band. From a computational point of view, a simple method is to compute the input signal power and compare it against a certain threshold. This threshold should be set according to the signal level in absence of the interference signal. Since the power of GNSS useful signal components at the receiver's antenna is extremely weak (several tens of $\mathrm{dB}$ below the background noise), the input signal power when the interference source is switched off is in practice the same as the background noise power, defined as

$$
\mathbb{E}\left\{\left|x_{i}[n]\right|^{2}\right\} \simeq \sigma^{2} .
$$

After the analog-to-digital converter (ADC) step, and exploiting the statistical properties of $\mathbf{n}$, it is possible to set a probability of false alarm, ie, the probability of detecting the interference even if the jamming signal is absent. Comparing the signal magnitude against the threshold and taking a decision in all the samples is not feasible in real time applications, so the detection algorithm runs on signal segments of $L$ samples. The estimated energy of a signal segment at the $i$ th antenna element is

$$
E_{i}=\sum_{k=1}^{L}\left|x_{i}[k]\right|^{2},
$$

where the random variable $\frac{E_{i}}{\sigma^{2}}$ follows a chi-square distribution with $2 L$ degrees of freedom. According to the tabulated values of such a distribution, it is possible to set the threshold with a certain probability of false alarm. When the segment's energy exceeds the detection threshold, the segment might contain an interference, and it is processed with the DOA estimation algorithm. Note that $\sigma^{2}$ should be estimated with a noise floor power estimation method. With the purpose of minimizing random effects, several noise power estimations are averaged on consecutive signal segments. In addition, as the receiver background noise may change over time, the estimation of $\sigma^{2}$ is performed periodically.

Considering a calibrated array, several algorithms can be used to estimate the interference signal DOA. In this contribution, we selected the well-known spectral Multiple Signal Classification (MUSIC) algorithm..$^{15, p .1158}$ The MUSIC test function can be defined as

$$
\hat{\theta}=\arg \max _{\theta} \frac{1}{\mathbf{v}(\theta)^{H}\left(\mathbf{I}-\mathbf{u} \mathbf{u}^{H}\right) \mathbf{v}(\theta)},
$$

where $\mathbf{v}(\theta) \in \mathbb{C}^{N \times 1}$ is the signal steering vector defined by its azimuth $\theta, \mathbf{u} \in \mathbb{C}^{N \times 1}$ is the eigenvector associated with the most powerful eigenvalue of $\hat{\mathbf{R}}_{\mathbf{x x}}=\frac{1}{K} \mathbf{X} \mathbf{X}^{H}$, and $\mathbf{I} \in \mathbb{R}^{N \times N}$ stands for the identity matrix.

Notice that in the experimental prototype described in Section 6, we have considered an array composed of $\mathrm{N}=2$ elements. As a consequence, only the interference azimuth, $\theta \in\left[0^{\circ}, 180^{\circ}\right)$, is considered, with an ambiguity of $180^{\circ}$.

Assuming the signal model defined in Equation 1, $\mathbb{E}\left\{\hat{\mathbf{R}}_{\mathbf{x x}}\right\}=\mathbf{U} \mathbf{\Lambda} \mathbf{U}^{H}$, where $\mathbf{U}$ is a unitary matrix whose columns are the eigenvectors of $\mathbf{R}_{\mathbf{x x}}$ and $\boldsymbol{\Lambda}=$ $\operatorname{diag}\left\{\lambda_{1}, \ldots, \lambda_{N}\right\}$, where $\lambda_{i}$ is defined as the corresponding eigenvalue. The most powerful eigenvalue and its eigenvector are associated with the interference subspace as follows:

$$
\begin{gathered}
\lambda_{\text {int }}=\mathbb{E}\left\{\frac{1}{K} \mathbf{d}_{\text {int }} \mathbf{d}_{\text {int }}^{H}\right\} \\
\mathbf{u}_{\text {int }}=\mathbf{h} .
\end{gathered}
$$

If there is only one interference impinging into the array, the rest of eigenvalues are $\lambda_{\text {noise }} \approx \sigma^{2}$. Besides the interference detection based on the energy estimation described in Equation 6, it is also possible to define a new test statistics based on the difference between eigenvalues as follows:

$$
\gamma_{\mathrm{dB}}=10 \log _{10}\left(\frac{\lambda_{\max }}{\lambda_{\min }}\right),
$$

where $\lambda_{\max }$ and $\lambda_{\min }$ stands for the maximum and minimum eigenvalues of $\hat{\mathbf{R}}_{\mathbf{x x}}$, respectively. If there is only one interference impinging into the array, then, $\gamma_{\mathrm{dB}}$ is an estimation of the interference-to-noise ratio (INR). 


\section{INTERFERENCE}

\section{LOCALIZATION ALGORITHM}

The bearings-only interference localization can be formulated as a maximum likelihood (ML) estimation problem. ${ }^{16}$ The goal is to obtain the ATC radar static position, $\mathbf{p}=$ $\left[p_{x}, p_{y}\right]^{\top}$ from a set of $M$ noisy estimated bearings, $\hat{\boldsymbol{\theta}}=$ $\left[\hat{\theta}_{1}, \ldots, \hat{\theta}_{M}\right]^{\top}$. The $i$ th estimated measurement $\hat{\theta}_{i}$ is computed at a sensor (array) known position, $\mathbf{r}_{i}=\left[r_{x, i} r_{y, i}\right]^{\top}$. Then the true bearing angle is defined as

$$
\theta_{i}(\mathbf{p})=\tan ^{-1}\left(\frac{p_{y}-r_{y, i}}{p_{x}-r_{x, i}}\right)
$$

and $\boldsymbol{\theta}(\mathbf{p})=\left[\theta_{1}(\mathbf{p}), \ldots, \theta_{M}(\mathbf{p})\right]^{\top}$. The ith estimated bearing-obtained from (7)—can be modeled as a noisy version of the true angle,

$$
\hat{\theta}_{i}=\theta_{i}+n_{i}, n_{i} \sim \mathcal{N}\left(0, \sigma_{n_{i}}^{2}\right),
$$

where the measurement noise is assumed to be Gaussian and independent across estimations. Considering the vector of $M$ estimated bearings $\hat{\theta}$, the $M \times M$ measurement error covariance matrix is $\mathbf{C}=\operatorname{diag}\left(\sigma_{n_{1}}^{2}, \ldots, \sigma_{n_{M}}^{2}\right)$, then the $\log$-likelihood function, $\ln f(\hat{\boldsymbol{\theta}} \mid \mathbf{q})$, can be written as

$$
-\frac{1}{2} \ln \left((2 \pi)^{M} \operatorname{det}(\mathbf{C})\right)-\frac{1}{2}(\hat{\boldsymbol{\theta}}-\boldsymbol{\theta}(\mathbf{q}))^{\top} \mathbf{C}^{-1}(\hat{\boldsymbol{\theta}}-\boldsymbol{\theta}(\mathbf{q}))
$$

where $\mathbf{q} \in \mathbb{R}^{2}$ represents the two-dimensional target location. Maximization of the log-likelihood function is equivalent to minimizing the cost function

$$
\begin{aligned}
J_{\mathrm{ML}}(\mathbf{q}) & =\frac{1}{2}(\hat{\boldsymbol{\theta}}-\boldsymbol{\theta}(\mathbf{q}))^{\top} \mathbf{C}^{-1}(\hat{\boldsymbol{\theta}}-\boldsymbol{\theta}(\mathbf{q})) \\
& =\sum_{i=1}^{M} \frac{1}{2 \sigma_{n_{i}}^{2}}\left(\hat{\theta}_{i}-\theta_{i}(\mathbf{q})\right)^{2} .
\end{aligned}
$$

The ML estimator of the ATC radar position is then given by

$$
\hat{\mathbf{p}}_{\mathrm{ML}}=\left.\arg \min _{\mathbf{q} \in \mathbb{R}^{2}} J_{\mathrm{ML}}(\mathbf{q}) \Rightarrow \frac{\partial J_{\mathrm{ML}}(\mathbf{q})}{\partial \mathbf{q}}\right|_{\mathbf{q}=\hat{\mathbf{p}}_{\mathrm{ML}}}=\mathbf{0},
$$

which is a nonlinear minimization problem and has no closed-form solution. We use an iterative procedure to optimize the cost function. The gradient of the cost function is given by

$$
\frac{\partial J_{\mathrm{ML}}(\mathbf{q})}{\partial \mathbf{q}}=\sum_{i=1}^{M} \frac{\theta_{i}(\mathbf{q})-\hat{\theta}_{i}}{\sigma_{n_{i}}^{2}\left\|\mathbf{q}-\mathbf{r}_{i}\right\|}\left[\begin{array}{c}
-\sin \left(\theta_{i}(\mathbf{q})\right) \\
\cos \left(\theta_{i}(\mathbf{q})\right)
\end{array}\right],
$$

and we can use a steepest-descent algorithm to recursively obtain the ML estimate of the radar position as

$$
\hat{\mathbf{p}}_{k+1}=\hat{\mathbf{p}}_{k}-\left.\mu \frac{\partial J_{\mathrm{ML}}(\mathbf{q})}{\partial \mathbf{q}}\right|_{\mathbf{q}=\hat{\mathbf{p}}_{k}},
$$

where $\hat{\mathbf{p}}_{0}$ is the radar position initialization and $\mu>0 \mathrm{a}$ small positive step size.

\section{6 | PROTOTYPE IMPLEMENTATION}

The idea of using extremely cheap RF front-ends to receive GNSS signals was already explored in Arribas et al, ${ }^{17}$ where the goal was to use a digital television front-end USB dongle to successfully obtain a position fix by adapting the open-source GNSS-SDR. The so-called RTLSDR receiver with a retail cost below US $\$ 25$ is based on Taiwan's Realtek RTL2832U DVB-T receiver chipset, sold in the form of USB dongles that allow users to watch over-the-air DVB-T European broadcast television on their personal computers (see Figure 12). Those devices send partially-decoded MPEG transport frames over the USB, but by exploiting an undocumented mode of operation of the demodulator chip, the user is able to obtain raw I\&Q samples, stream them through USB to a personal computer and then apply the GNSS-SDR software processing. ${ }^{17}$

At that time, in September 2013, Juha Vierinen, a researcher involved in the Kilpisjärvi Atmospheric Imaging Receiver Array (KAIRA), Finland, did an experiment with two RTLSDR's dongles, modified for sharing the same reference clock source. ${ }^{18}$ The hardware modification consisted of removing the $28.8 \mathrm{MHz}$ XTAL in one of the dongles (see Figure 12) and connecting the clock input directly to the other dongle XTAL signal output. Apparently, the two front-ends are synchronized in both the downconversion chain and in the baseband sampling process. However, due $t$ the independent USB transport streams and different sampling start times, both sample streams are not synchronized as seen from the SDR software running in the CPU, resulting in a time shift between channels, constant in time during the capture or processing session but random over sessions.

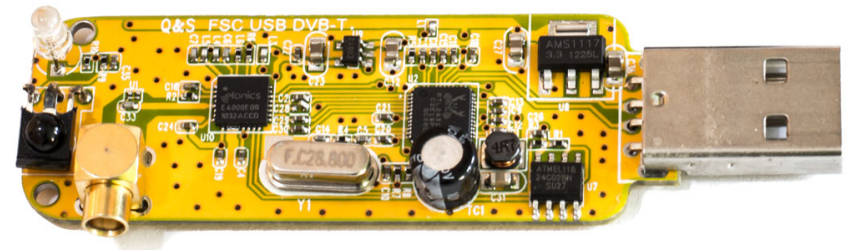

FIGURE 12 RTLSDR dongle with onboard reference clock [Color figure can be viewed at wileyonlinelibrary.com and www.ion.org] 


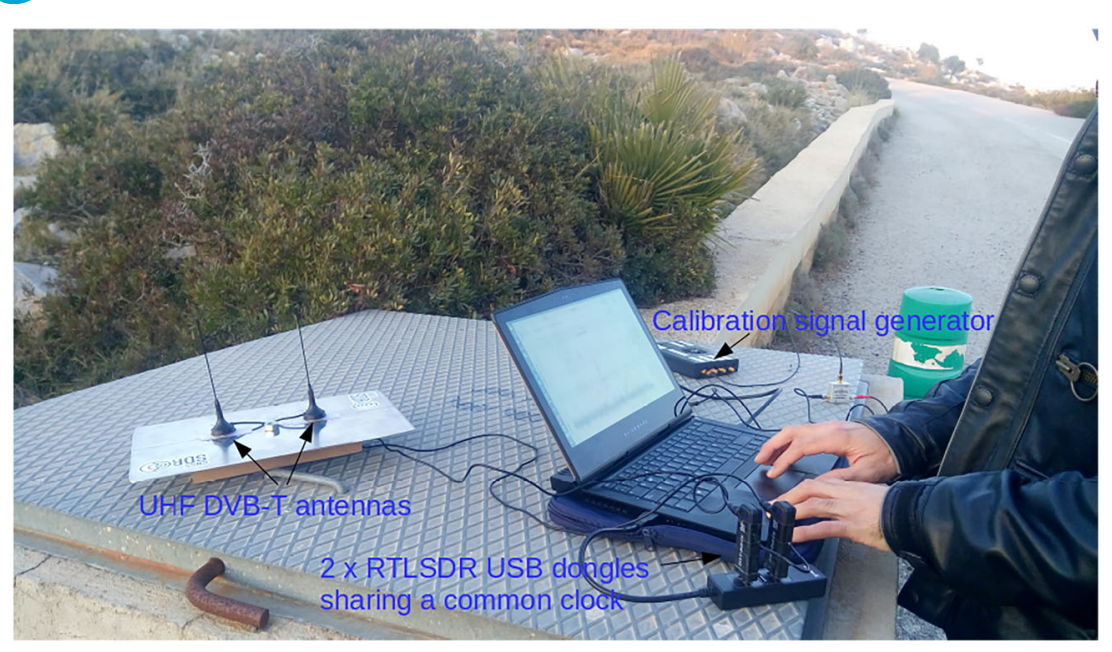

FIGURE 13 Ultra low cost antenna array prototype with $\mathrm{N}=2$ elements [Color figure can be viewed at wileyonlinelibrary.com and www.ion.org]

Considering the array signal model in Equation 1, it is possible to model the RTLSDR sample stream misalignment as follows:

$\mathbf{X}(t)=\left[\begin{array}{ccc}x_{1}\left(t-(K-1) T_{s}\right) & \ldots & x_{1}(t) \\ \vdots & \ddots & \vdots \\ x_{N}\left(t-(K-1) T_{s}+\Delta T_{s t r_{N}}\right) & \ldots & x_{N}\left(t+\Delta T_{s t r_{N}}\right)\end{array}\right]$

where $\Delta T_{s t r_{i}}=K_{s t r_{i}} T_{s}$ is the random time delay present in the $i$ th array channel equivalent to an integer number of samples $K_{\text {str }}$.

Some time later, in 2015, Piotr Krysik, who wanted to use a set of RTLSDRs to receive multiple GSM frequency channels at a time, published a GNU Radio Companion (GRC) block $^{19}$ that effectively performs a time synchronization that estimates $\Delta T_{s t r_{N}}$ by automating the following steps:

1. Tune the RTLSDR dongles to the same frequency where some transmission or calibration signal is present.

2. Record short signals with all of the dongles.

3. Compute a cross-correlation of the signals (ie, with respect to one selected channel), finding position of maximums of cross-correlations in order to estimate relative delays of the channels.

4. Correct the delays so the channels are time-synchronized.

5. Switch the dongles to their target frequencies, changing other parameters of the channels to the target values.

Figure 13 shows a picture of the complete implementation of the ultra low-cost GNSS interference detector and localization. From left to right, an $\mathrm{N}=2$ element array, with $\frac{\lambda}{2}$ separation between elements is built with two regular UHF antenna monopoles that come with the DVB-T dongles attached to an aluminum ground plane. Next, two RTLSDR dongles featuring the common clock modification are connected to the antenna array outputs by equal length RF cables. A regular laptop attached to a USB 3.0 hub drives the dongles with the SDR software components, described hereafter.

For the sake of simplicity in this experiment, the calibration signal source is obtained using a USRP B210 and a two-port splitter which is manually connected to the RTLSDR inputs in each operation. In a fully automatic

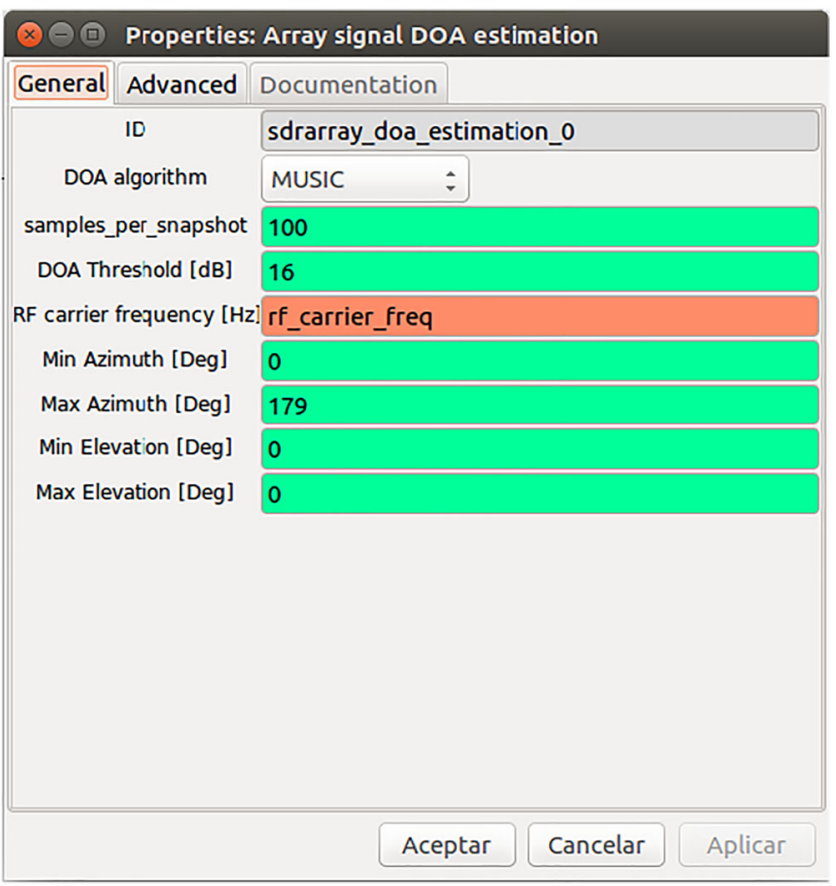

FIGURE 14 GNU Radio Companion custom array direction of arrival (DOA) estimation block [Color figure can be viewed at wileyonlinelibrary.com and www.ion.org] 


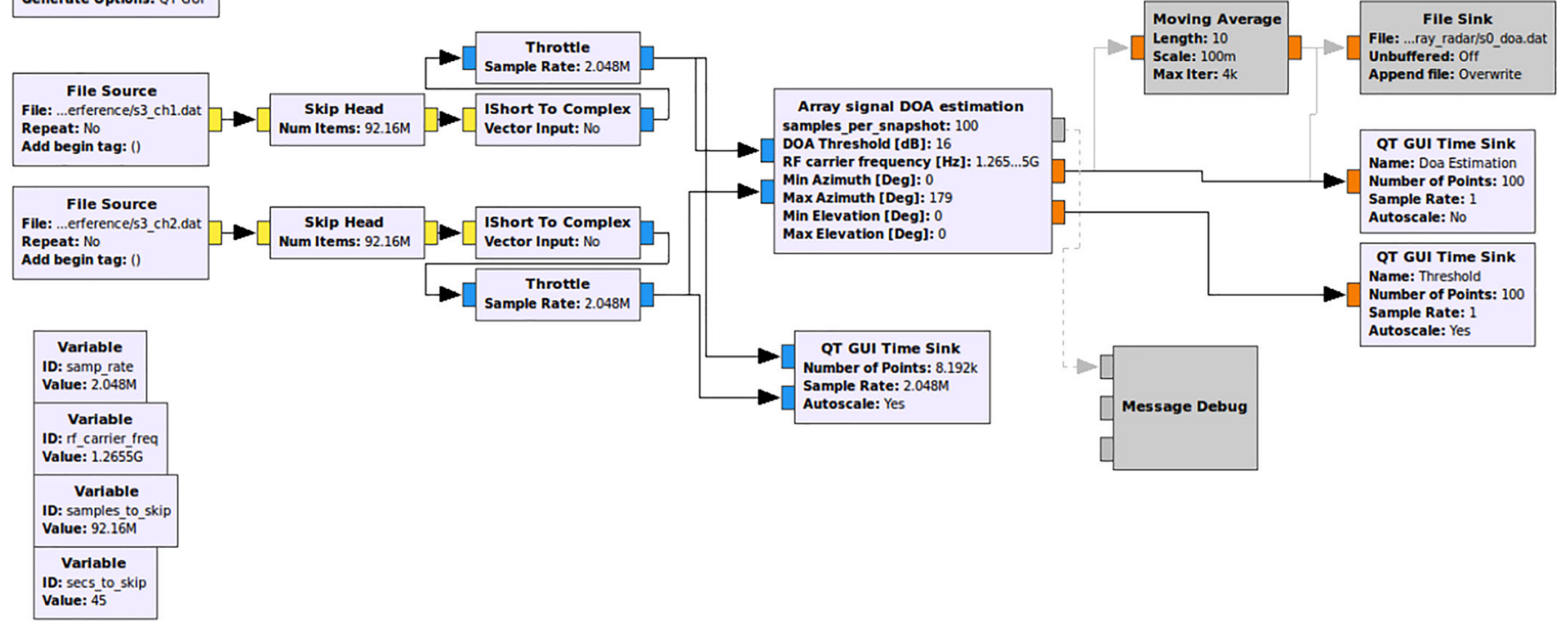

FIGURE 15 GNU Radio Companion direction of arrival (DOA) estimation block diagram for post-processing [Color figure can be viewed at wileyonlinelibrary.com and www.ion.org]
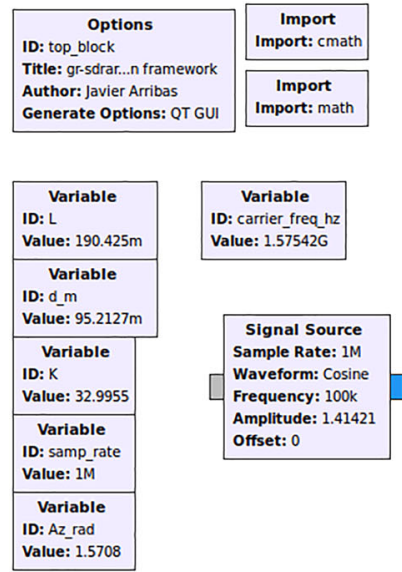

\begin{tabular}{|l|l|}
\hline \multicolumn{1}{|c|}{ QT GUI Range } & \multicolumn{1}{c|}{ QT GUI Range } \\
ID: Az_deg & ID: SNR_db \\
Label: DOA Azimuth [de & Label: INR [dB] \\
Default Value: 90 & Default Value: 6 \\
Start: 0 & Start: -20 \\
Stop: 180 & Stop: 20 \\
Step: 1 & Step: 1 \\
\hline
\end{tabular}
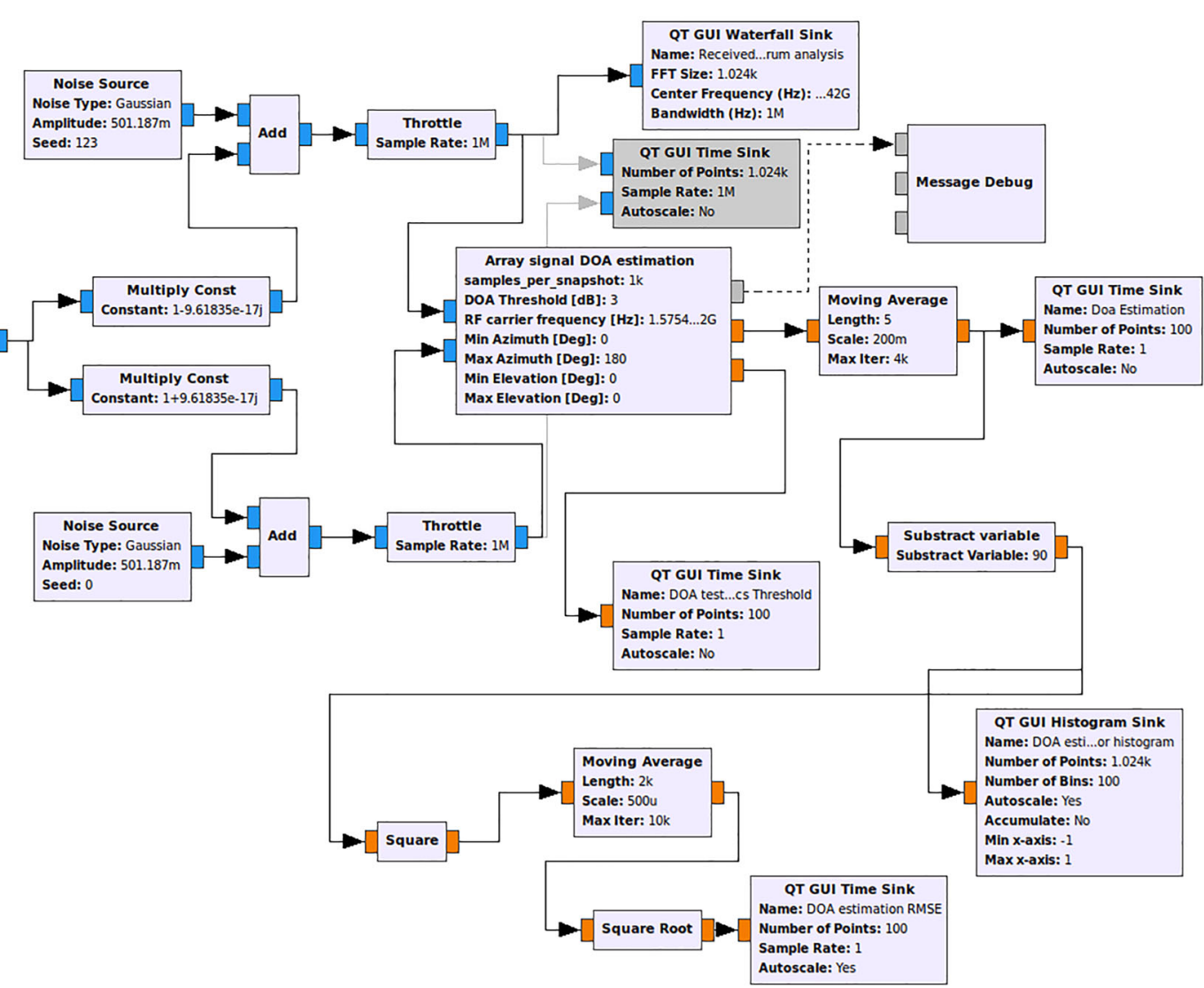

FIGURE 16 GNU Radio Companion direction of arrival (DOA) estimation block diagram for performance evaluation by simulations [Color figure can be viewed at wileyonlinelibrary.com and www.ion.org]

prototype, the calibration signal could be easily implemented by an analog noise source (see, eg, Maxim Integrated ${ }^{20}$ ) and a two-port coupler, built in the same PCB.
The approximated overall hardware cost of the prototype, excluding the laptop, but including the noise generator and the coupler, is below US \$ 50 . 
The array signal processing and the DOA estimation algorithm are implemented using the GNU Radio SDR framework as a custom out-of-tree block library named gr-sdrarray, freely available under the GPL v3 license in Arribas. ${ }^{21}$ Figure 14 shows a screen capture of the GNU Radio Companion DOA estimation block interface.

The user options are:

- DOA algorithm selection: currently, the MUSIC algorithm described in Section 4 and a simple phased array beamforming sweep are implemented.

- Samples per snapshot: sets the number of array samples used in each DOA estimation (defined as $K$ in Equation 1).

- DOA threshold $[d B]$ : sets the minimum test statistic value to trigger a positive DOA estimation. If the MUSIC algorithm is selected, the test statistic is defined as the ratio between the maximum and minimum eigenvalues expressed in $\mathrm{dB}$. If the beamforming sweep is selected, the test statistic is the ratio between the maximum and minimum array output power for the complete direction sweep expressed in $\mathrm{dB}$.

- $R F$ center frequency [Hz]: defines the array center frequency and sets the separation between elements to $\frac{\lambda}{2}$. Currently, the number of array elements is set to 2 .

- Min, Max Azimuth and Min, Max Elevation [deg]: defines the DOA exploration space.

The block outputs are:

- DOA estimation [deg]: synchronous GNU Radio output port (float data type) containing DOA estimations when the test statistic is above the configured threshold.

- DOA estimation [deg]: asynchronous message output port containing DOA estimations when the test statistic is above the configured threshold.

- Test statistic [dB]: synchronous GNU Radio output port (float data type) containing the test statistic value for each valid DOA estimation.

The DOA estimation block can work either in real time or in post processing. In order to assess different DOA

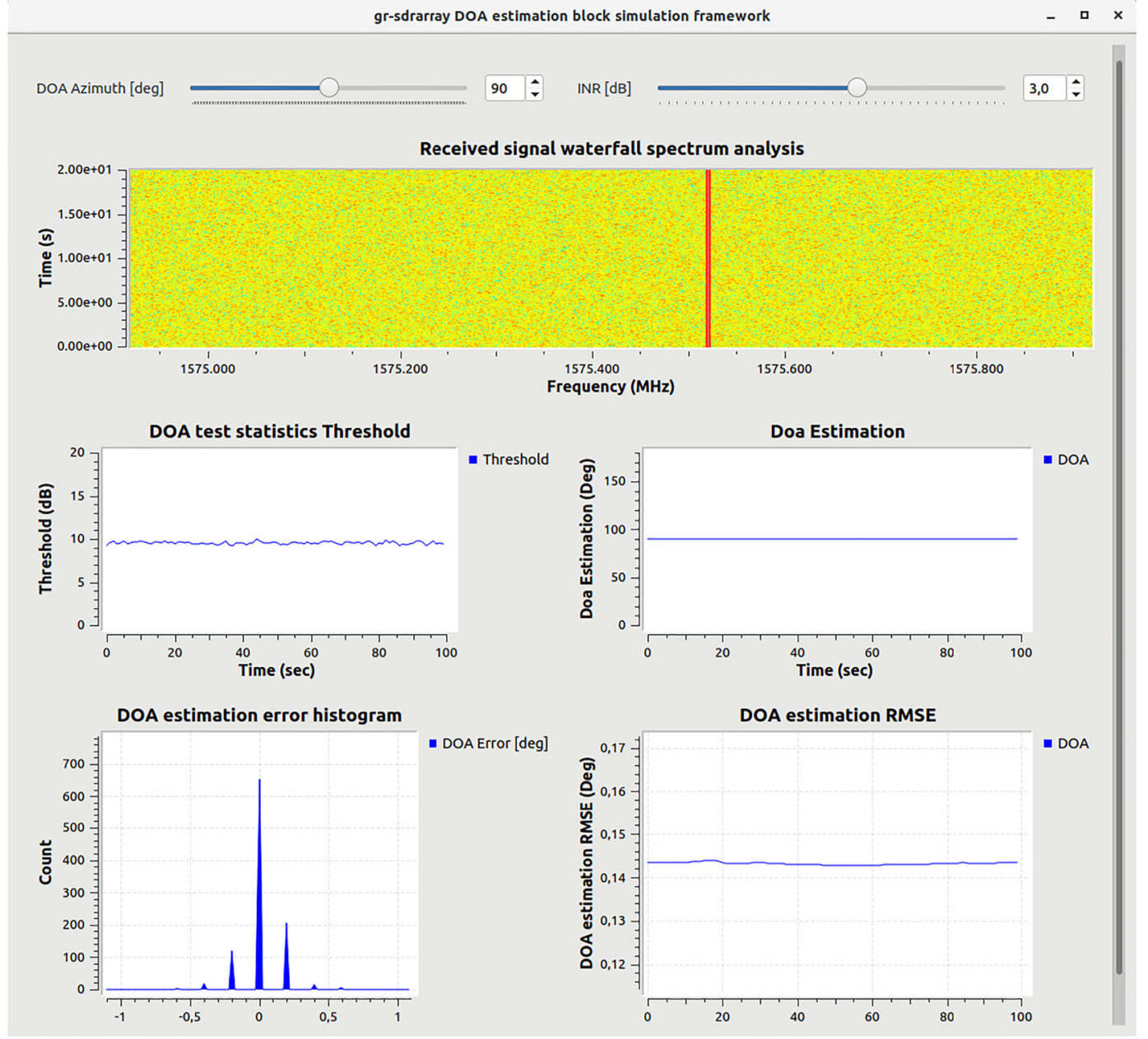

FIGURE 17 DOA estimation performance under simulated interference, DOA Az $=90$ Deg, INR $=3 \mathrm{~dB}$ [Color figure can be viewed at wileyonlinelibrary.com and www.ion.org] 


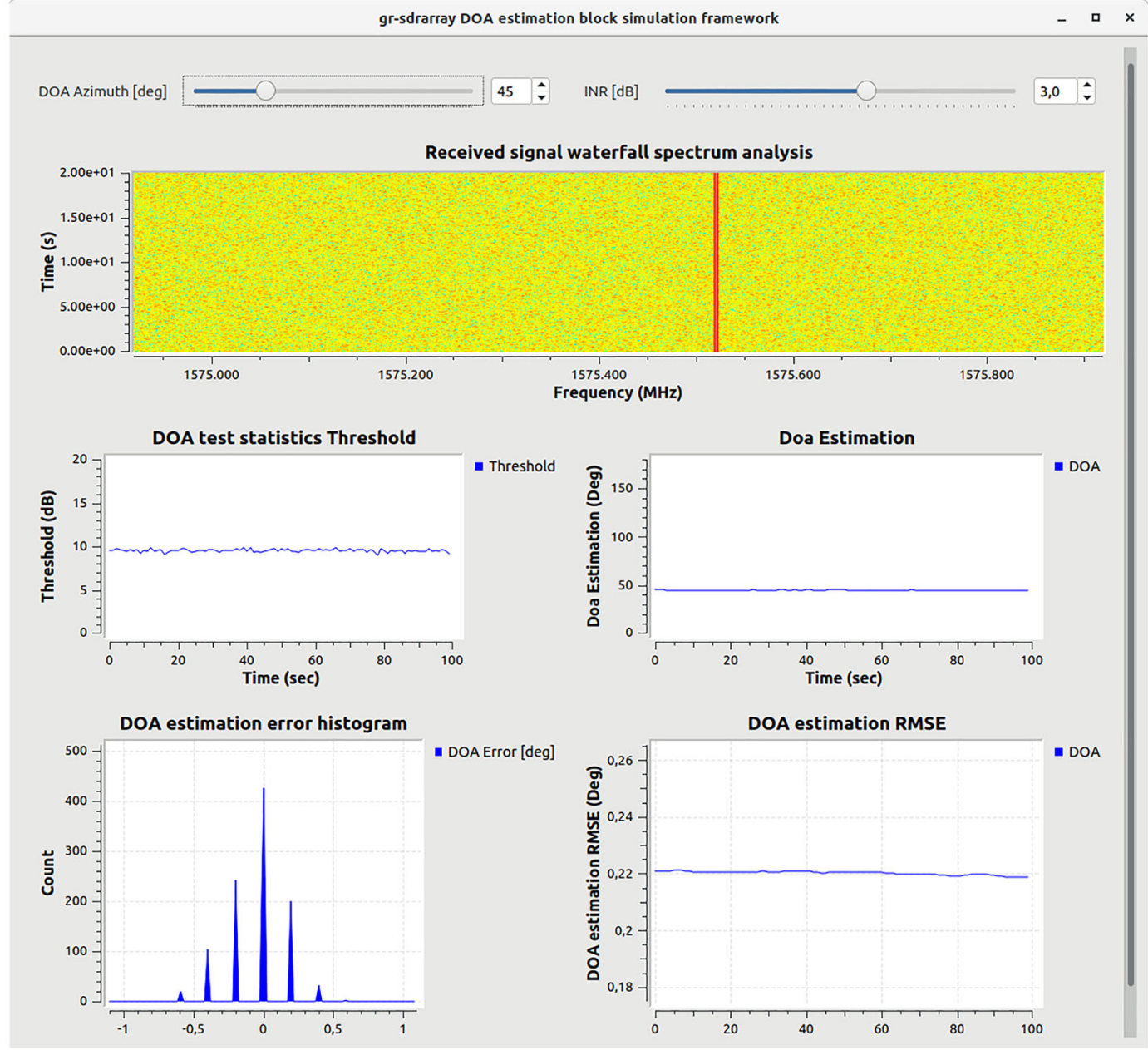

FIGURE 18 DOA estimation performance under simulated interference, DOA Az $=45$ Deg, INR $=3 \mathrm{~dB}$ [Color figure can be viewed at wileyonlinelibrary.com and www.ion.org]

estimation algorithms and thresholds in the same signal, we captured the array baseband samples in files for post-processing. Figure 15 shows the GNU Radio Companion block diagram for DOA estimation in post processing. From left to right, the samples are loaded from file sources (skipping the first 45 seconds to exclude the calibration signal transitory). The array snapshots are fed to the DOA estimation block. Its output ports are connected to QT time sinks for visualization. In addition, the DOA estimations are saved into files by using a file sink.

\section{7 | ALGORITHM VALIDATION WITH SIMULATED SIGNALS}

In order to validate the algorithm implementation and to assess the DOA estimation performance, a simulation framework was created consisting of a set of GNU Radio blocks and the GNU Radio companion tool. Figure 16 shows a block diagram of the simulation framework. Compared with Figure 15, the signal source file has been replaced by a waveform generator configured to generate a single tone (in the testing, the tone is shifted $100 \mathrm{kHz}$ from the GPS L1 central frequency and the simulation sampling frequency was set to $1 \mathrm{MHz}$ ). Next, two Multiply Const. are in charge of producing the corresponding phase shifts for the two antennas according to an adjustable DOA. The simulator can control the INR and two independent Gaussian noise generators (Noise Source in the diagram) are in charge of contaminating the impinging signal of both antennas. Finally, the Array signal DOA estimation block receives the two simulated inputs and produces the DOA estimation output and their associated detection threshold. The data is presented to the user in real-time plots. The DOA estimation error is also computed in real-time by subtracting the true DOA data provided by the user input to the estimated DOA and performing both histogram and root mean square error (RMSE) plots.

The user interface is shown in Figures 17 through 20. These figures are snapshots of the results for INRs of 3,0 , and $-6 \mathrm{~dB}$, respectively. In Figure 17, the interfer- 


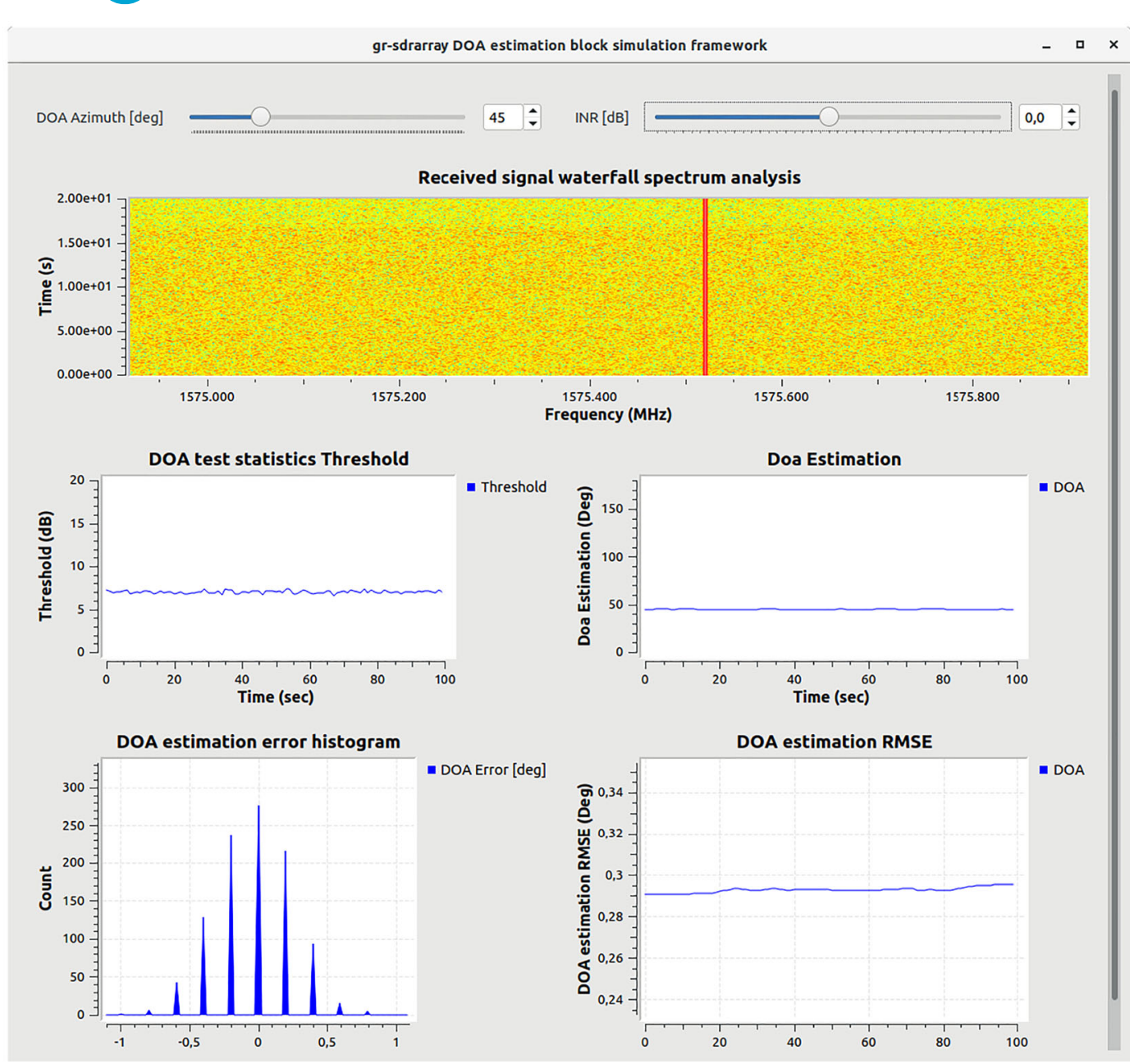

FIGURE 19 DOA estimation performance under simulated interference, DOA Az $=45$ Deg, INR $=0 \mathrm{~dB}$ [Color figure can be viewed at wileyonlinelibrary.com and www.ion.org]

ence DOA azimuth was set to $90^{\circ}$, impinging the array from the broadside with an INR of $3 \mathrm{~dB}$. In such a favorable condition, the array is able to estimate the DOA with an RMSE of $0.145^{\circ}$. In Figures 18-20, the DOA azimuth was set to $45^{\circ}$. The results show that, under Gaussian noise, the DOA estimation algorithm is unbiased and its RMSE increases as the interference losses power. Recalling the inherent protection of GNSS signals against interferences, thanks to their spreading gain, the degradation for lower INR values might not be considered an issue in practice. Nevertheless, with the proposed methodology, an interference of $6 \mathrm{~dB}$ below the noise floor, impinging the array with $45^{\circ}$ of azimuth, was detected and its DOA estimated with an RMSE error less than $1^{\circ}$.

\section{MEASUREMENT CAMPAIGN RESULTS}

On February 23, 2018, the authors demonstrated the capability of the low-cost interference localization prototype by performing a measurement campaign in the surroundings of the Barcelona ATC radar, located in the Garraf Natural Park. Figure 21 shows the locations of the measurement points (P1 to P6) and the radar location. In each measurement point, the array was deployed as shown in Figure 22. The array was oriented with the broadside pointing to the geographic North in all the measurements. The nearest point (P2) is $108 \mathrm{~m}$ away from the interference source and the furthest measurement point (P6) is located $1101 \mathrm{~m}$ away from the interference.

A DOA estimation result sample is shown in Figure 23, where the GNU Radio Companion flow graph is run in post-processing for a measurement recorded in P4. From top to bottom, the first time evolution plot shows the test statistics, the second plot is the DOA estimation and the third plot is the signal amplitudes (I and Q) for the two antenna elements. The sampling frequency was set to 2.048 MSPS and the RTLSDR automatic gain control was enabled in order to avoid signal saturation. The radar pulses are clearly visible well above the noise floor, thus 


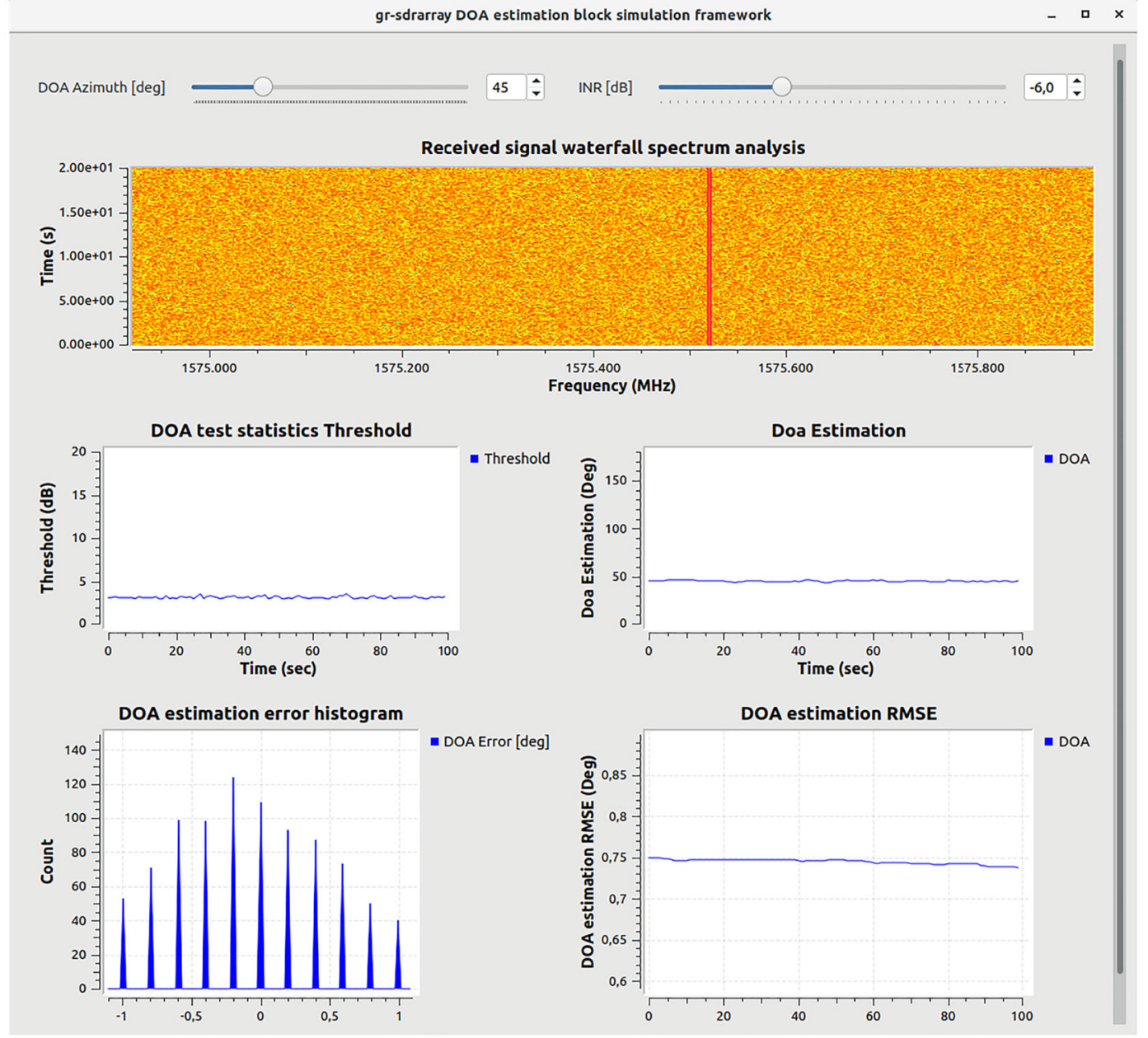

FIGURE 20 DOA estimation performance under simulated interference, DOA Az $=45 \mathrm{Deg}$, INR $=-6 \mathrm{~dB}$ [Color figure can be viewed at wileyonlinelibrary.com and www.ion.org]

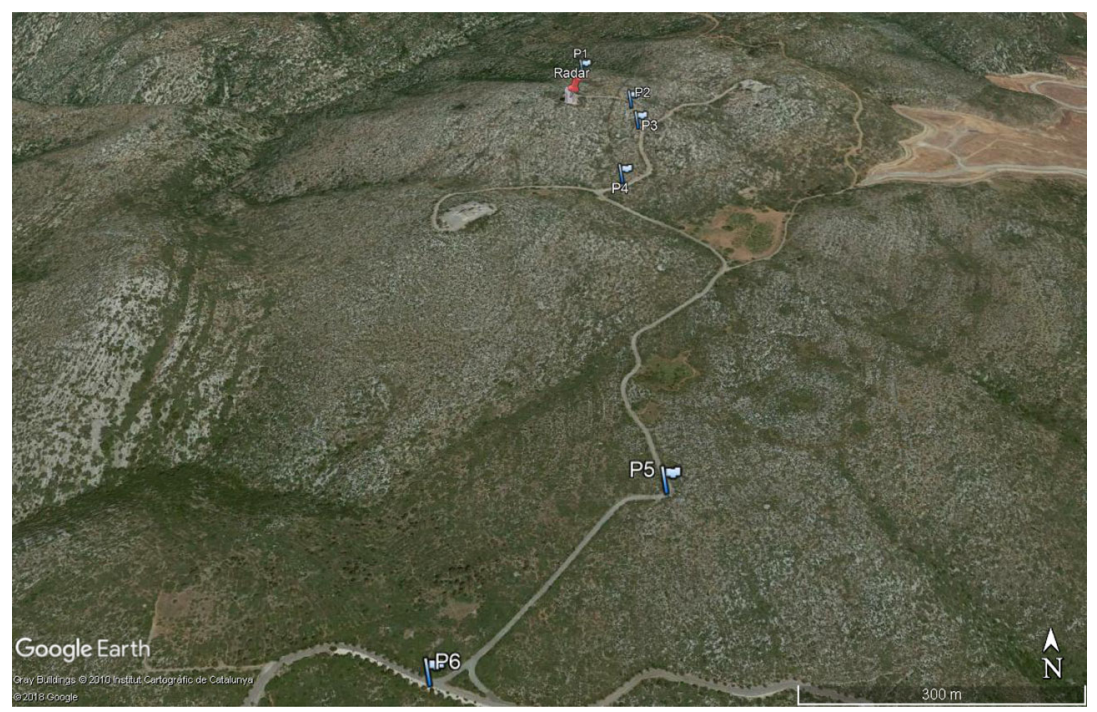

FIGURE 21 Direction of arrival (DOA) measurement waypoints [Color figure can be viewed at wileyonlinelibrary.com and www.ion.org]

the DOA estimation shows quite stable values. This procedure was performed for all the measurement points and the DOA estimations were stored in files.
The DOA estimations were compared with the true DOA values obtained from the ATC radar true coordinates in order to evaluate the estimation accuracy of the system. 


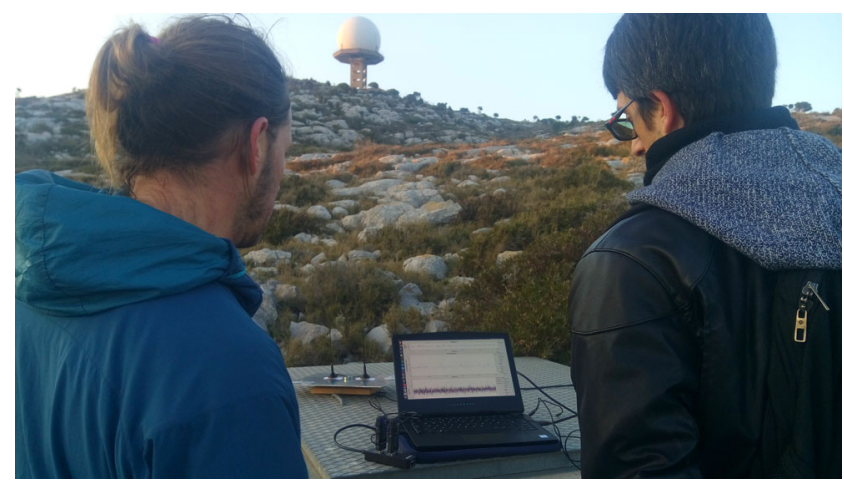

FIGURE 22 Prototype placed at measurement point P3 [Color figure can be viewed at wileyonlinelibrary.com and www.ion.org]

Figure 24 shows the performance of the DOA estimations at measurement point $\mathrm{P} 5$, smoothed with a 10-point moving average. It can be seen that the estimated DOA is stable in time, with some outliers and a bias of approximately $7^{\circ}$. Considering the simulation results shown in Section 7, the DOA estimation bias present in the measurement campaign may be caused mainly by an error in the array bearing, which was set to point the array broadside to the true geographic North using a regular smartphone compass application.

Finally, the triangulation algorithm was implemented in a MATLAB script that reads the DOA estimation files and

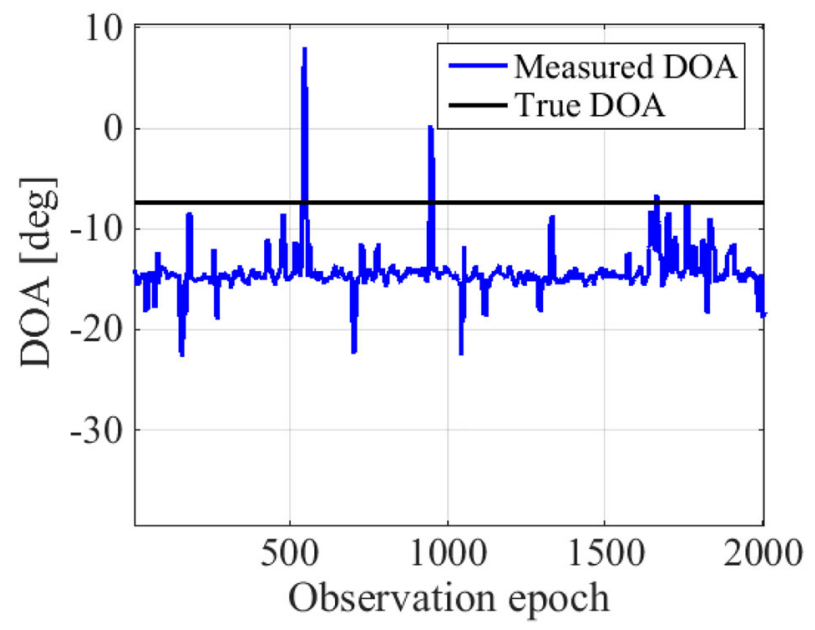

FIGURE 24 Interference direction of arrival (DOA) estimation measured in measurement point P5 [Color figure can be viewed at wileyonlinelibrary.com and www.ion.org]

uses the measurement point coordinates to perform the steepest-descent solver described in Equation 17.

The initial interference position estimation $\hat{\mathbf{p}}_{0}$ was set to an arbitrary value located in the surrounding measurement points. The number of steps was set to 10000 and the step size was set to $\mu=0.0001$.

Figure 25 shows the evolution of the position estimation for each step. The final position estimation error is below $60 \mathrm{~m}$.

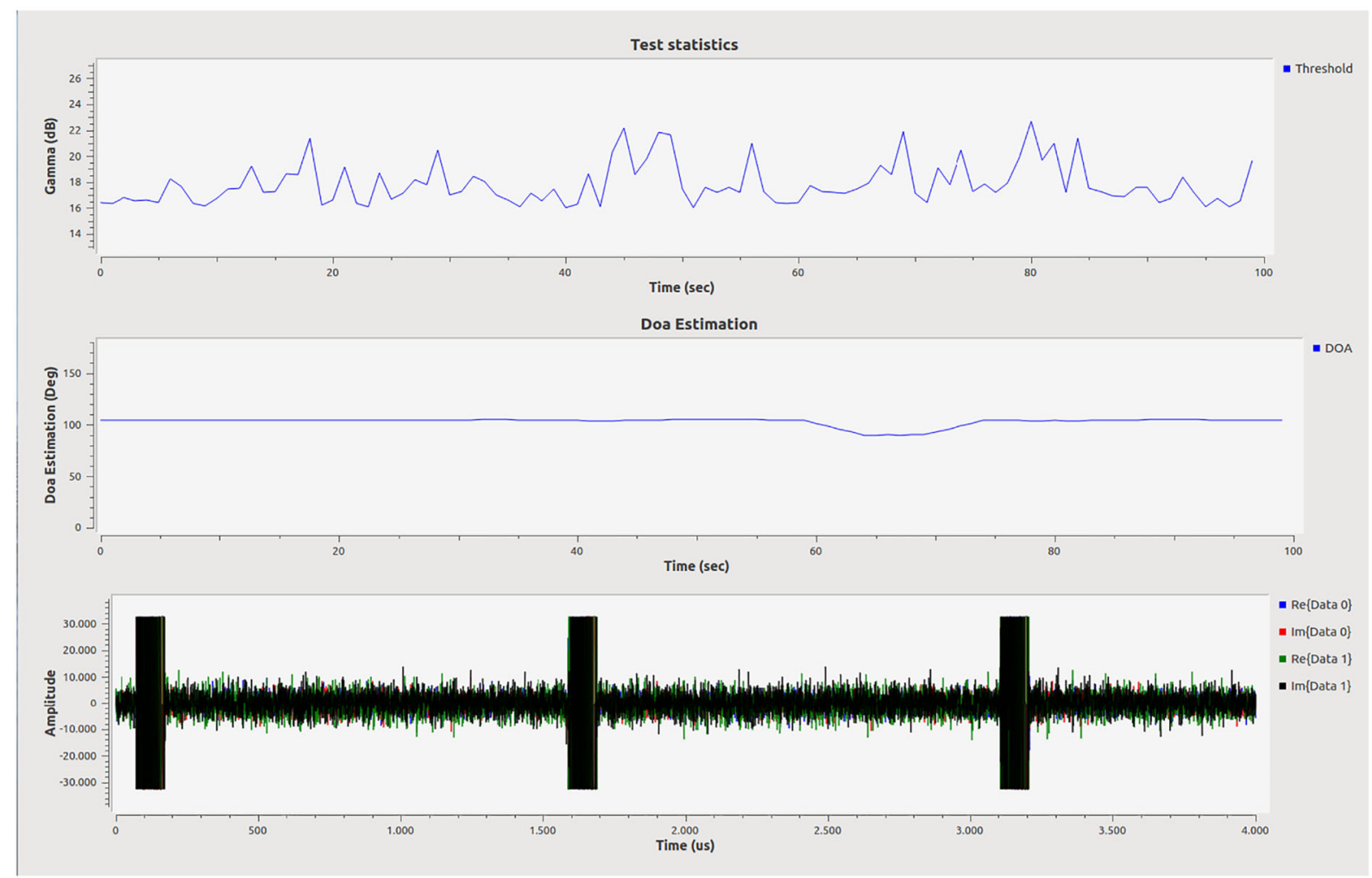

FIGURE 23 Screenshot of the GNU Radio Companion direction of arrival (DOA) estimation block running in post-processing for DOA measurement point P4 [Color figure can be viewed at wileyonlinelibrary.com and www.ion.org] 
6. European GNSS Agency. Galileo Commercial Service Implementing Decision enters into force, GSA Today Newsletter, 2017. https://www.gsa.europa.eu/newsroom/news/galileocommercial-service-implementing-decision-enters-force

7. International Telecommunications Union (ITU). Geneva. ITU Radio Regulations 2016, 2016, http://www.itu.int

8. European Conference of Postal and Telecommunications Administrations (CEPT) Working Group Frequency Management, European Common Allocation (ECA). European Table of Frequency Allocations in the Range $8.3 \mathrm{kHz}$ to $3000 \mathrm{GHz}$; June 2016.

9. Borio D. Swept GNSS jamming mitigation through pulse blanking. In: Proceedings of the European Navigation Conference $(E N C)$; June 2016; Helsinki, Finland:1-8. https://doi.org/10. 1109/EURONAV.2016.7530549

10. Arribas J, Ramos A, Fernández-Prades C, Vilà-Valls J, Closas P. Air traffic radar interference event in the Galileo E6 band: detection, analysis and mitigation. In: Proceedings of the 30th International Technical Meeting of the Satellite Division of the Institute of Navigation (ION GNSS+ 2017); September 2017; Portland, OR:1204-1228.

11. GNSS-SDR: An open source Global Navigation Satellite Systems software-defined receiver. Website accessed on January 11, 2019, Online: https://gnss-sdr.org

12. Arribas J, Fernández-Prades C, Closas P. GESTALT: A testbed for experimentation and validation of GNSS software receivers. In: Proceedings of the 28th International Technical Meeting of the Satellite Division of the Institute of Navigation (ION GNSS+ 2015); September 2015; Tampa, FL:3222-3234.

13. Analog Devices. AD9361 RF Agile Transceiver. http://www. analog.com/en/products/rf-microwave/integrated-transceivers -transmitters-receivers/wideband-transceivers-ic/ad9361.html; September 2017.

14. Tsui JB-Y. Fundamentals of Global Positioning System Receivers. A Software Approach. John Wiley \& Sons, Inc.; New York: 2000.
15. Van Trees HL. Optimum Array Processing. John Wiley \& Sons; New York: 2002.

16. Donğançay K. Bearings-only target localization using total least squares. Signal Process. 2005;85(9):1695-1710.

17. Arribas J, Fernández-Prades C, Closas P. Multi-antenna techniques for interference mitigation in GNSS signal acquisition. EURASIP J Adv Signal Process. 2013;2013(1):143. https://doi. org/10.1186/1687-6180-2013-143

18. Vierinen J. KAIRA: The Kilpisjärvi Atmospheric Imaging Receiver Array. http://kaira.sgo.fi/2013/09/ 16-dual-channel-coherent-digital.html

19. Krysik P. Multi-channel receiver with use of RTL-SDR dongles. https://github.com/ptrkrysik/multi-rtl

20. Maxim Integrated. Application Note 3469: Building a Low-Cost White-Noise Generator. March 2005. Available at https:// www.maximintegrated.com/en/app-notes/index.mvp/id/3469. Accessed: January 11, 2019.

21. Arribas J. A GNURadio blocks library for SDR array signal processing. https://bitbucket.org/jarribas/gr-sdrarray; 2018.

How to cite this article: Arribas $\mathrm{J}$, Vilà-Valls $\mathrm{J}$, Ramos A, Fernández-Prades C, Closas P. Air traffic control radar interference event in the Galileo E6 band: Detection and localization. NAVIGATION. 2019;66:505-522. https://doi.org/10.1002/navi.310 